\title{
Modell 9+3 der SAMK: \\ Interessant für Militärärzte in ziviler Weiter- bildung, Spitaldirektionen und Klinikchefärzte
}

H. Bürgi, S. Bankoul

Korrespondenz:

Direktion SAMK

Logistikbasis der Armee Sanität

Worblentalstrasse 36

CH-3063 Ittigen

Tel. 0313242723

Fax 0313237197

peter.kehl@vtg.admin.ch
Die Schweizerische Integrierte Akademie für Militär- und Katastrophenmedizin (Projekt SAMK) bietet Militärärzten eine grosse Zahl von Fortbildungskursen an, die auch zivil anerkannt werden. Daneben erleichtert das Modell 9+3 der SAMK aber auch jungen Militärärzten die zivile Weiterbildung, indem es militärdienstlich bedingte $\mathrm{Ab}$ wesenheiten teilweise kompensiert.

Das Modell 9+3 ist für eidgenössisch diplomierte Ärzte und Ärztinnen bestimmt, die zum Leutnant der Sanitätstruppen oder des Rotkreuzdienstes brevetiert wurden und die den 12 wöchigen praktischen Dienst als Militärarzt (Abverdienen des Offiziersgrades) geleistet haben. Von diesem Angebot kann der Militärarzt nur einmal in seiner Laufbahn Gebrauch machen.

Der Arzt sucht sich selbst eine mindestens einjährige Weiterbildungsstelle als Assistenzarzt an einer Klink, die mindestens für ein Jahr Weiterbildung anerkannt ist. Erfüllt eine Weiterbildungsstelle diese Bedingungen nicht, oder ist das Spital nicht Mitglied von H+ Spitäler Schweiz (z.B. Grundlageninstitute), entscheidet die Direktion SAMK von Fall zu Fall über die Anerkennung als Modell 9+3.

Die letzten drei Monate (89 Tage) dieses Ausbildungsjahres gelten als Grundausbildungsdienst (GAD) für Offiziere (fakultatives «Praktikum» mit Anrechnung an die Gesamtdienstleistungspflicht). Die Weiterbildung ist identisch mit der von der FMH anerkannten der betreffen- den Klinik, d.h. der Militärarzt leistet seinen Dienst in Zivil und untersteht für seine Spitalarbeit dem Klinikchefarzt. Das Zivilspital hat während dieser Zeit Anspruch auf die Erwerbsausfallentschädigung, spart also einen erheblichen Teil des Salärs.

\section{An Militärärzte (Offiziere)}

Die Sanität in der Logistikbasis der Armee offeriert Ihnen im Rahmen des Projektes Schweizerische integrierte Akademie für Militär- und Katastrophenmedizin (SAMK) die folgenden Weiter- oder Fortbildungskurse, die zum grossen Teil schon durch die FMH anerkannt sind und die auch an Ihre jährliche Militärdienstpflicht angerechnet werden.

Interessenten wollen sich bitte rechtzeitig anmelden: www.vbs-ddps.ch/internet/groupgst/ de/home/sanit/wehrmedizin.html.

\section{Aux médecins militaires (officiers)}

L'élément sanitaire de la base logistique de l'armée vous offre, dans le cadre du projet Académie suisse intégrée de médecine militaire et de catastrophe (ASIMC), les cours suivants de formation postgraduée ou continue, déjà en grande partie reconnus par la FMH et imputables à votre service militaire annuel obligatoire.

Les intéressés voudront bien s'annoncer à temps: www.vbs-ddps.ch/internet/groupgst/de/ home/sanit/wehrmedizin.html. 


\begin{tabular}{|c|c|c|}
\hline \multicolumn{2}{|l|}{ Kursangebote der SAMK / Offres des cours ASIMC } & \\
\hline \multicolumn{3}{|l|}{ Anästhesie und Reanimation in Basel } \\
\hline \multicolumn{3}{|l|}{ Kurssprache: Deutsch und/oder Englisch } \\
\hline Anästhesiekongress * & 17./18.3.2006 & BS 1 \\
\hline Notarztkurs * & $23 . / 24.3 .+30 . / 31.3 .2006$ & BS 2 \\
\hline Notarztkurs * & $16 . / 17.11+23 . / 24.11 .2006$ & BS 3 \\
\hline ACLS * & $30 . / 31.5 .2006$ & BS 4 \\
\hline ACLS * & 10./11.10.2006 & BS 5 \\
\hline ATLS Student Course, deutsch/englisch * & 20.-22.3.2006 & BS 6 \\
\hline ATLS-Student Course, deutsch/englisch * & 13.-15.9.2006 & BS 7 \\
\hline PALS * & 17./18.8.2006 & BS 9 \\
\hline Prehospital Airway Management * & 19.1.2006 & BS 10 \\
\hline Prehospital Airway Management & 9.11 .2006 & BS 11 \\
\hline Advanced Complication Management * & 10.1 .2006 & BS 12 \\
\hline Advanced Complication Management & 21.11 .2006 & BS 13 \\
\hline \multicolumn{3}{|l|}{ Zahnmedizin und Kieferchirurgie in Basel } \\
\hline \multicolumn{3}{|l|}{ Kurssprache: Deutsch und/oder Englisch } \\
\hline $\begin{array}{l}\text { Bernd-Spiessl-Symposium für innovative und visionäre } \\
\text { Technologien, englisch }\end{array}$ & 16.-18.6.2006 & ZM 1 \\
\hline Traumatologie des Kopfes, deutsch & 22.-24.11.2006 & $\mathrm{ZM} 2$ \\
\hline \multicolumn{3}{|c|}{ Médecine et management en cas de catastrophe et de guerre à Lausanne } \\
\hline \multicolumn{3}{|l|}{ Cours en langue française ou allemande } \\
\hline $\begin{array}{l}\text { Principes de médecine de catastrophe (risques, concepts } \\
\text { d'intervention, terminologie) * }\end{array}$ & 27./28.1.2006 & VD 1-1 [M1] \\
\hline $\begin{array}{l}\text { Principes de médecine de catastrophe (risques, concepts } \\
\text { d'intervention, terminologie) }\end{array}$ & 14./15.9.2006 & VD 1-2 [M1] \\
\hline $\begin{array}{l}\text { Catastrophe à effets limités (accidents majeurs) pour } \\
\text { professionnels de la chaîne des urgences. } \\
\text { Formation prérequise: VD } 1\end{array}$ & 11.-13.5.2006 & VD 2 [M2] \\
\hline $\begin{array}{l}\text { Catastrophe à effets limités (accidents majeurs) } \\
\text { cours avancé de conduite pour chefs des secours } \\
\text { (médecins et ambulanciers). Formation prérequise: } \\
\text { VD } 1 \text { + VD } 2\end{array}$ & 15./16.6.2006 & VD $3[\mathrm{M} 3]$ \\
\hline $\begin{array}{l}\text { Sanitätsdienstliche Führung Grossereignis (SFG) } \\
\text { (Teil A: «Knowledge», Grundlagen, Prinzipien, Partner). } \\
\text { Voraussetzung: keine }\end{array}$ & 3. -5.5 .2006 & VD A [SFG-A] \\
\hline $\begin{array}{l}\text { Sanitätsdienstliche Führung Grossereignis (SFG) } \\
\text { (Teil B: «Training», Kommunikation und Teaminteraktion). } \\
\text { Voraussetzung: VD A [SFG-A] }\end{array}$ & 23./24.11.2006 & VD B [SFG-B] \\
\hline \multicolumn{3}{|l|}{ Chirurgie à Genève } \\
\hline \multicolumn{3}{|l|}{ Cours en langue française } \\
\hline Cours pratique de chirurgie de guerre et de catastrophe & 7./8.9.2006 & GE 1 \\
\hline ATLS Student Course, français/anglais & 23.-25.10.2006 & GE 2 \\
\hline \multicolumn{3}{|l|}{ Innere Medizin, Infektiologie in Bern } \\
\hline \multicolumn{3}{|l|}{ Kurssprache: Deutsch } \\
\hline 4. Systematisches Infektiologiecurriculum & 8.11 .2006 & BE $1 \mathrm{~A}$ \\
\hline 9. Berner Infektiologiesymposium & 9.11 .2006 & BE 1B \\
\hline $\begin{array}{l}\text { 1. Systematisches Curriculum Internmedizinische } \\
\text { Notfallmedizin }\end{array}$ & 8.9.2006 & BE $2 A$ \\
\hline 5. Berner Notfallsymposium & 7.9 .2006 & BE 2 B \\
\hline
\end{tabular}




\begin{tabular}{|c|c|c|}
\hline $\begin{array}{l}\text { Kursangebote der SAMK / Offres des cours } \\
{ }^{*}=\text { Kurse bereits ausgebucht }\end{array}$ & & \\
\hline Innere Medizin, Infektiologie in Bern (Fortsetzung) & & \\
\hline Kurssprache: Deutsch & & \\
\hline Notarztkurs & 16./17.11.+23./24.11.2006 & BE 3 \\
\hline ATLS Student Course, deutsch/englisch * & 16. -18.10 .2006 & BE 4 \\
\hline Notarztkurs & 22.-25.3.2006 & BE 5 \\
\hline Katastrophen- und Wehrpsychiatrie in Zürich & & \\
\hline Kurssprache: Deutsch & & \\
\hline $\begin{array}{l}\text { Psychologische Nothilfe (PNH) in der Armee: } \\
\text { eine Managementaufgabe }\end{array}$ & 18.-20.10.2006 & $\mathrm{ZH} 1$ \\
\hline Einführungskurs in die PNH nach Richtlinien NNPN & 18. -20.10 .2006 & $\mathrm{ZH} 2$ \\
\hline ATLS Student Course, deutsch/englisch * & 11./12.9.2006 & $\mathrm{ZH} 3$ \\
\hline PHTLS, Grundausbildungskurs, deutsch/englisch & 7./8.3.2006 & $\mathrm{ZH} 4$ \\
\hline Centre spécialisé pour soins, Lausanne & & \\
\hline Cours en langue française & & \\
\hline Hygiène et sanitation & 15.-17.2.2006 & SM 1 \\
\hline Soins physique & 29. -31.3 .2006 & SM 2 \\
\hline Approches interculturelles/risques/sécurité/ressources & 21.-23.9.2006 & SM 3 \\
\hline Aspects psychologiques & 25. -27.10 .2006 & SM 4 \\
\hline L'expatrié dans sa mission & 7.-9.12.2006 & SM 5 \\
\hline Transfusionswesen, Lugano & & \\
\hline Kurssprache: Deutsch/Französisch & & \\
\hline Katastrophe und Transfusion von Blutprodukten & 9.6 .2006 & LU 1 \\
\hline Kontaktadresse / Adresse de contact & & \\
\hline $\begin{array}{l}\text { LBA - Sanität, Direktion SAMK, Worblentalstrasse 36, } \\
\text { Internet: www.vbs-ddps.ch/internet/groupgst/de/hon }\end{array}$ & $\begin{array}{l}\text { en, Tel. } 03132427 \text { 23, Fax C } \\
\text { rehrmedizin/kurse.html }\end{array}$ & 197 \\
\hline
\end{tabular}

